\title{
Jejunal perforation due to ingested buffalo bone mimicking acute appendicitis
}

\author{
Ajay Kumar Yadav* , Gyanendra Malla ${ }^{1}$, Kunal Bikram Deo², Saroj Giri ${ }^{1}$, Bishnu Murti Bhattarai² \\ and Shailesh Adhikary ${ }^{2}$
}

\begin{abstract}
Background: Foreign body ingestion is seen quite frequently in clinical practice, intestinal perforation due to this is rare. The foreign body often mimics another cause of acute abdomen and requires an emergency surgical intervention. The majority of patients do not recall ingesting sharp foreign bodies.

Case presentation: We report an interesting case of a fifty five year-old man who presented with pain in the right iliac fossa with localised tenderness which was clinically diagnosed as acute appendicitis. During the operation, the presence of purulent collection and the inflamed bowel with flakes raised suspicion of bowel perforation. The assessment of the proximal small bowel revealed two small perforations in the jejunum. A hard, bony and sharp object was extracted and the perforations were closed. Post-operative recovery was uneventful. Detailed food history was taken following the recovery of the patient from surgery. It revealed the history ingestion of home prepared buffalo meat. The extracted object was identified as 'buffalo bone' by the patient and the care taker of the patient. The jejunum was perforated by the ingested buffalo bone causing local peritonitis in right iliac fossa.
\end{abstract}

Conclusion: Intestinal perforation by ingested foreign bodies should be suspected in acute abdomen. It requires a high degree of suspicion and awareness on the part of the clinician.

Keywords: Local peritonitis, Jejunum perforation, Foreign body, Buffalo bone, Case report

\section{Background}

The majority of ingested foreign bodies (IFB) are excreted from the digestive tract without any complications or morbidity; however, occasionally they may lead to serious clinical problems, such as obstruction, perforation or bleeding [1-3]. Although IFB are quite common problem in children, they are infrequently encountered in adults but are seen in elderly people wearing dentures, alcoholics and/or patients with learning difficulties [4]. IFB, such as chicken bones, fish bones, toothpicks and dentures require surgical intervention in $5 \%$ of cases. Patients are not usually aware of the IFB which is usually detected either during laparotomy or at the time of pathological examination of the surgical specimen [5]. Less than $1 \%$ of IFB, especially large, sharp and/or pointed objects, cause

\footnotetext{
*Correspondence: ajay.yadav@bpkihs.edu

${ }^{1}$ Department of General Practice and Emergency Medicine, B. P. Koirala Institute of Health Sciences, Dharan, Nepal

Full list of author information is available at the end of the article
}

bowel perforation. Perforation usually occurs at the narrowest part of the bowel, either at the ileocecal valve or at the rectosigmoid junction [6]. Sharp fish bone causing perforation in the duodenum has been reported [7]. This case report highlights the importance of detailed history taking for food ingestion in a case presenting with symptoms of acute appendicitis where the pain characteristics are not very typical of appendicitis.

\section{Case presentation}

A fifty five year-old man presented to the emergency room (ER) with a localized pain at the right iliac fossa. There were no histories of nausea, vomiting or any other complaints associated with the pain. History taking revealed that the pain was colicky in nature around the epigastric region when it started 3 days ago. The pain increased gradually by the next day, which eventually localized at the right iliac fossa before the case presented to our ER. 
The pain at the right iliac fossa was a continuous type which was aggravated by any kind of movement and was relieved by rest. The patient did not give any past history of medical or surgical problems. There were no known drug allergies or use of any medications. He was afebrile. Abdominal examination revealed localised tenderness and with guarding in the right iliac fossa. The baseline work up was normal. The appendix was not visualized on ultrasonogram but revealed minimal fluid collection in the right iliac fossa. A diagnosis of acute appendicitis was done and a plan for emergency appendectomy was made. During operation, Gridiron incision was made to visualize the appendix, which was mildly inflamed. On cut section, the mucosa was found to be inflamed and the presence of a faecolith was noted. Following appendectomy, the presence of inflamed bowel with food flakes and purulent collection raised suspicion of intestinal perforation. We decided to explore the entire bowel. The incision was converted to Rutherford Morrison incision. Bowel examination revealed two small jejunal perforations around $2 \mathrm{~mm}$ size in the antimesenteric border, approximately $250 \mathrm{~cm}$ proximal to ileocaecal junction. On palpation of the adjacent bowel, a hard, bony and sharp object was felt. This object was removed through the perforation site. The perforations were repaired in two layers with $3 / 0$ polygalactin and $3 / 0$ silk sutures. A pelvic drain was placed, after which the abdomen was closed in layers. Postoperative recovery was uneventful. Histopathology report of the appendix revealed eosinophils, which indicated receding appendicitis. The extracted sharp and bony foreign body was discussed among the surgical team as a sharp piece of animal bone. After patient recovery, both the patient and caretaker were interviewed in detail about the food history before the pain started. The history revealed the ingestion of home cooked buffalo meat with alcohol in the evening by the patient. The patient usually consumed buffalo meat with alcohol in the evenings. The object was confirmed as a 'buffalo bone' by both the patient and the caretaker (Figs. 1, 2).

\section{Discussion}

The diagnosis of the patient based on the history, clinical examination and laboratory investigation initially pointed towards acute appendicitis. The symptoms were not typical only of acute appendicitis; however, due to lack of enough evidence, other differential diagnosis could not be considered immediately. Retrospectively, the pain can be discussed to be caused primarily by the progression of the perforation. The released intestinal contents may have collected in the right iliac fossa. This could have inflamed the appendix causing the symptoms that mimic appendicitis. A variety of foreign bodies such

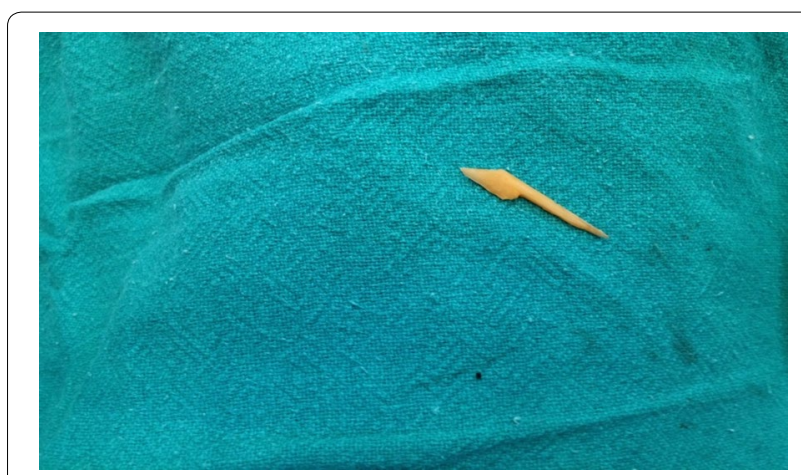

Fig. 1 The foreign body: a buffalo bone

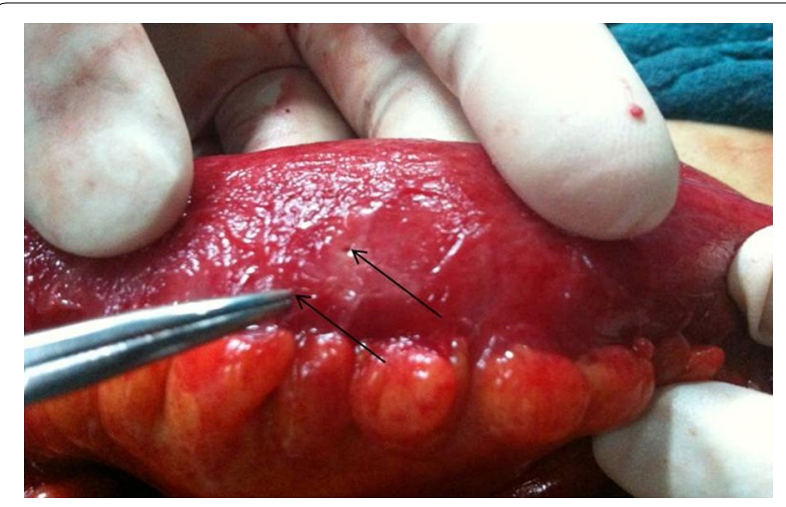

Fig. 2 Two tiny jejunal perforations (indicated by the tip of the arrows)

as dentures, toothpicks, chicken or fish bone and cocktail sticks have been implicated in the pathogenesis of gastrointestinal perforation [8]. Perforations are common in elderly individuals (who wear dentures), alcoholics, children and mentally challenged individuals. Less than $1 \%$ of ingested foreign bodies perforate the bowel, and the greatest risk is with large, sharp or pointed objects. The foreign bodies usually pass through the esophagus without any complications, there is an increased risk for bowel perforations, bowel obstruction and other complications of complications [9]. Most common site for intestinal perforation is terminal ileum and rectosigmoid region [10]. In our patient perforation was at jejunum. The patient did not provide a history of ingestion of a foreign body, his admission to the hospital was due to a severe lower abdominal pain and we did not observe subdiaphragatic free air on a radiological evaluation. Patients often do not recall ingesting foreign body. The foreign body is found either during investigations or during surgery. Patients may present with abdominal pain, nausea, vomiting, fever, haematochezia or melena. Foreign-body perforation of the bowel presents as acute abdominal emergency mimicking acute appendicitis, diverticulitis 
or perforated peptic ulcer [11]. Free air is seen in $15.9 \%$ of gastrointestinal perforations in the subdiaphragmatic region [10]. The foreign material perforates the intestinal wall and an inflammation occurs in this region with a granulation tissue covering intestinal wall and omentum with fibrin material. This can explain the low rate of subdiaphragmatic free air in a direct radiography [12]. Hard triangular plastic material was recovered during exploration laparotomy in jejunum perforation [13]. In a case of 3-months history of abdominal pain, fever and leucocytosis, an explorative laparotomy revealed fish bone. The case had jejunal perforation due to the fish bone [14]. A fish bone perforation mimicking acute appendicitis is also reported [15]. While fish bones are sharp and perforations are reported $[14,15]$, the case presented here had two small jejunal perforations due to a buffalo bone. $\mathrm{He}$ presented with symptoms of acute appendicitis. Perforations were repaired primarily (Additional file 1).

\section{Conclusions}

Intestinal perforation by ingested foreign bodies should be suspected in acute abdomen. A detailed food history may be required for cases presenting with symptoms of acute appendicitis that do not have a typical pain history of appendicitis. It requires a high degree of suspicion and awareness on the part of the clinician.

\section{Additional file}

Additional file 1. Care case report timeline.

\section{Abbreviations}

IFB: ingested foreign bodies; ER: emergency room.

\section{Authors' contributions}

$A K Y, S G, G M$, were involved in managing the case at the Emergency Ward. KBD, BMB and SA performed surgery. AKY, GM, KBD, SG, BMB and SA contributed to the conception of the case report. Preliminary report was prepared by $A K Y, K B D$ and SA. The draft was revised and inputs were given by all authors. Literature review was performed by AKY, GM and SA. All authors read and approved the final manuscript.

\section{Author details}

${ }^{1}$ Department of General Practice and Emergency Medicine, B.P. Koirala Institute of Health Sciences, Dharan, Nepal. ${ }^{2}$ Department of General Surgery, B.P. Koirala Institute of Health Sciences, Dharan, Nepal.

\section{Acknowledgements}

The authors would like to acknowledge the contribution from Dr. Shyam Sundar Budhathoki, Assistant Professor, School of Public Health and
Community Medicine, B. P. Koirala Institute of Health Sciences for contributing during the manuscript preparation and proof reading the manuscript draft.

\section{Competing interests}

The authors declare that they have no competing interests.

Availability of data and materials

All available data are included within the manuscript.

\section{Consent for publication}

Written informed consent was obtained from the patient for publication of this Case report and any accompanying images.

Received: 13 February 2016 Accepted: 16 June 2016

Published online: 24 June 2016

\section{References}

1. Paul RI, Christoffel KK, Binns HJ, Jaffe DM. Foreign body ingestions in children: risk of complication varies with site of initial health care contact. Pediatric Practice Research Group. Pediatrics. 1993;91:121-7.

2. Hashmonai M, Kaufman T, Schramek A. Silent perforations of the stomach and duodenum by needles. Arch Surg. 1978:113:1406-9.

3. Cheng W, Tam PK. Foreign-body ingestion in children: experience with 1265 cases. J Pediatr Surg. 1999;34:1472-6.

4. Rodríguez-Hermosa Jl, Codina-Cazador A, Sirvent JM, Martín A, Gironès J, Garsot E. Surgically treated perforations of the gastrointestinal tract caused by ingested foreign bodies. Colorectal Dis. 2008;10:701-7.

5. Yilmaz M, Akbulut S, Ozdemir F, Gozeneli O, Baskiran A, Yilmaz S. A swallowed dental prosthesis causing duodenal obstruction in a patient with schizophrenia: description of a new technique. Int J Surg Case Rep. 2012;3:308-10.

6. Kornprat P, Langner C, Mohadjer D, J Mischinger K. Chicken-bone perforation of a sigmoid colon diverticulum into the right groin and subsequent phlegmonous inflammation of the abdominal wall. Wien Klin Wochenschr. 2009:121:220-2.

7. Umemoto T, Harada Y, Sakata M, Yokomizo K, Kigawa G, Nemoto H, Hibi K. A case of duodenal perforation due to ingested fish bone revealed by computed tomography. Nihon Gekakei Rengo Gakkaishi (Journal of Japanese College of Surgeons). 2013;38(4):806-8.

8. Schwartz GF, Polsky HS. Ingested foreign bodies of the gastrointestinal tract. Am Surg. 1976;42:236-8.

9. Theodoropoulou A, Roussomostakaki M, Michalodimitrakis MN, Kanaki C, Kouroumalis EA. Fatal hepatic abscess caused by a fishbone. Lancet. 2002;359:977.

10. Goh B, Chow P, Quah H, Ong H, Eu K, Ooi L, et al. Perforation of the gastrointestinal tract secondary to ingestion of foreign bodies. World J Surg. 2006;30:372-7.

11. Pinero Madrona A, Fernandez Hernandez JA, Carrasco Prats M, Riquelme Riquelme J, Parrila Paricio P, et al. Intestinal perforation by foreign bodies. Eur J Surg. 2000;166:307-9.

12. Yagmur $\mathrm{Y}$, Ozturk H, Ozturk H. Distal ileal perforation secondary to ingested foreign bodies. J Coll Physicians Surg Pak. 2009;19:452-3.

13. Colak B, Yuce Y. Jejunum perforation with a foreign body: case report. Research. 2014;1:936.

14. Sofo L, Princi P, Piraino A, Caprino P. Jejunal perforation by a foreign body. Internet J Surg. 2008;21:2.

15. Almoudaris AM, Chow A, Kaneria S, Jiyad Z, Hadjiminas DJ. Fish bone perforation mimicking acute appendicitis. J Med Cases. 2011;2(6):296-9. 